\section{La muerte no respeta festivos}

\section{Death does not respect Holidays}

\section{Señor Editor,}

La mayor esperanza de vida de la población, que se espera siga aumentando, es el logro más importante de la humanidad. Este "triunfo demográfico" es un gran desafío para las sociedades debido a que implica una serie de cambios sociales, culturales y, por cierto, económicos y políticos. Un aspecto central en este aumento de la longevidad tiene que ver con que la mayor cantidad de años vividos no necesariamente implica una extensión del tiempo de vida saludable. De hecho, evidencias internacionales y también nacionales demuestran que junto con el incremento de la esperanza de vida aumentó el tiempo de vida con enfermedades crónicas, siendo más aguda esta situación entre las mujeres ${ }^{1,2}$. Además, se ha constatado una rectangularización de la curva de sobrevida en Chile, junto con un desplazamiento hacia la derecha como consecuencia del aumento de la edad modal de muerte ${ }^{3}$. Ante ese escenario es que mediante la presente carta quiero llamar la atención respecto de la inexistencia de una política y/o programa integral de cuidados paliativos en Chile focalizada hacia aquellos adultos mayores (AM) que se encuentran en la etapa final de su vida.

En términos conceptuales, por cuidados paliativos se entiende a aquellos que buscan mejorar la calidad de vida de los pacientes y sus familias que se enfrentan con enfermedades amenazantes para la vida, que pueden provocar la muerte, mediante el tratamiento del dolor y otros síntomas angustiantes ${ }^{4}$. El avance en este tipo de políticas de salud pública en Chile no es algo reciente, por ejemplo, en 1990 se inició la prestación de cuidados paliativos domiciliarios, mientras que en 1995 se publicó la Norma Alivio Dolor y Cuidados Paliativos $^{5}$. Posteriormente, el año 2003 el Gobierno chileno incluyó este tipo de cuidados en el sistema de atención pública a través de las Garantías Explícitas de Salud (GES). No obstante, todas estas iniciativas han estado focalizadas en las enfermedades oncológicas, dejando de lado todas aquellas otras dolencias que de manera similar a los cánceres, deterioran la calidad de vida y salud, no sólo del paciente que está en su etapa final de la vida, sino que también de la familia y, en especial, de los cuidadores del paciente.

Como un ejemplo de lo descrito es posible mencionar el caso de SPI, una anciana de 90 años que falleció el 12 de octubre del 2020. Ella estaba inscrita en el "Programa Alivio al Dolor" implementado por la Dirección de Salud Municipal de la comuna de Colina, Región Metropolitana, Chile. Sin embargo, la cobertura del programa no incluye sábados, domingos y/o días festivos, por lo cual, esta adulta mayor falleció postrada en su cama, luego de agonizar durante todo el fin de semana "largo" sin recibir los cuidados paliativos que su caso ameritaba. Este caso, ciertamente que es un hecho que, con escaso margen de error, se repite en diversas comunas y regiones del país y, refleja una falencia del sistema de salud nacional que podría ser suplida a través de una norma de cuidados paliativos integral.

Tal como el título sugiere, "la muerte no respeta festivos", resulta incomprensible e inhumano desde un enfoque de derechos, que una anciana postrada no pueda acceder a una atención que contribuya a la disminución de sus dolores durante sus últimas horas de vida debido a que el programa no tiene cobertura los fines de semana o festivos.

El Gobierno chileno en el año 2017 ratificó la Convención Interamericana sobre la Protección de los Derechos Humanos de las Personas Mayores que insta a los Estados para que adopten medidas adecuadas con la finalidad de que los AM que reciban cuidados de largo plazo cuenten "con servicios de cuidados paliativos que abarquen al paciente, su entorno y su familia" 6 . Por lo cual, no contar con una ley o política nacional de cuidados paliativos integral es incumplir con los propios compromisos asumidos como gobierno. Desde esta perspectiva, no sólo es un deber político que nuestro país cuente con una ley de este tipo, sino que también es un deber ético, moral y social.

Ciertamente, la promulgación de una ley "universal o nacional" de cuidados paliativos permitirá que Chile avance en respetar y promover la calidad de vida de los adultos mayores otorgando respuesta a todas las necesidades físicas, emocionales, espirituales y sociales de los pacientes y sus respectivas familias, incluyendo los/as cuidadores/as. Chile, como un país que avanza en políticas sanitarias y que históricamente ha estado a la vanguardia en la implementación de políticas sociales y de salud pública, debe reconocer que democratizar los cuidados paliativos es el camino correcto que permitirá garantizar la dignidad de los adultos mayores, cuyo estado de salud esté deteriorado por enfermedades crónicas o terminal (más allá del cáncer) y, de esta forma aminorar el sufrimiento y dolor asociado.

El envejecimiento poblacional en nuestro país como resultado de la transición demográfica y el aumento de las expectativas de vida en todas las edades (siendo inclusive significativo el incremento de la esperanza de vida entre los octogenarios y nonagenarios), sumado a que la prolongación de la vida se ha acompañado por el cambio en el perfil de enfermedades -donde actualmente existe una alta prevalencia de enfermedades crónicas y degenerativas-hace sumamente necesaria una política que dignifique el morir. En la medida en que aumente la proporción de adultos mayores y la edad de muerte modal siga desplazándose hacia la derecha es relevante una política de cuidados paliativos que contribuya a 
sobrellevar los últimos momentos de vida de los adultos mayores. En especial, de aquellos de bajos recursos socioeconómicos que presenten dificultades para acceder a programas del sector privado.

Agradecimientos: El autor agradece el soporte entregado por el programa PAI Convocatoria Nacional Subvención a Instalación en la Academia Convocatoria Año 2019, No 77190035. Sin embargo, se deja de manifiesto que las opiniones vertidas acá son de exclusiva responsabilidad del autor.

Moisés H. Sandoval ${ }^{1, a}$ ${ }^{1}$ Departamento de Nutrición Pública, Instituto de Nutrición y Tecnología de los Alimentos (INTA), Universidad de Chile. aPhD. en Demografía.

\section{Referencias}

1. Moreno X, Lera L, Albala C. Disability-free life expectancy and life expectancy in good self-rated health in Chile: Gender differences and compression of morbidity between 2009 and 2016. PLoS One [Internet]. 2020; 15 (4): 1-12. Available from: http://dx.doi.org/10.1371/journal.pone.0232445.

2. Guedes GR, Castro M, Camargos S, Cristina P, Siviero L, Machado CJ, et al. Educational and sex differences in the functional disability life expectancy for the elderly: Brazil,
1998 and 2003. Cad Saúde Coletiva [Internet]. 2011; 19 (2): 187-96. Available from: https://www.academia.edu/973808/ Approximating_the_educational_differences_in_mortality_demographic_indirect_techniques\%0A.

3. Zepeda Ortega A, Monteverde L M. Compresión de la mortalidad en Chile: 1969-2002. Pap Ppoblac [online]. 2016; 22 (87): 265-91.

4. OMS | Cuidados paliativos [Internet]. [cited 2020 Nov 6]. Available from: https://www.who.int/cancer/palliative/es/

5. Pastrana T, De Lima L, Wenk R, Eisenchlas J, Monti C, Rocafort J, Centeno C. Atlas de Cuidados Paliativos de Latinoamérica ALCP. $1^{\text {a }}$ edición. Houston: IAHPC Press. Available from: https://cuidadospaliativos.org/uploads/2012/10/ atlas/Atlas\%20de\%20Cuidados\%20Paliativos\%20en\%20 Latinoamerica.pdf.

6. OEA - Organización de los Estados Americanos. Convención Interamericana sobre la Protección de los Derechos Humanos de las Personas Mayores [Internet]. 2015. Available from: http://www.oas.org/es/sla/ddi/docs/tratados_multilaterales_interamericanos_A-70_derechos_humanos_personas_mayores.pdf.

Correspondencia a:

Moisés H. Sandoval

Instituto de Nutrición y Tecnología de los Alimentos, INTA,

Universidad de Chile, Avenida El Líbano, 5524, Macul. Santiago, Chile.

msandoval@inta.uchile.cl 